Monatsschr Kinderheilkd 2010 • 158:118-119 DOI 10.1007/s00112-010-2164-x

Online publiziert: 28. Januar 2010

(c) Springer-Verlag 2010

F. Zepp

Zentrum für Kinder- und Jugendmedizin, Johannes Gutenberg-Universität Mainz

\title{
Gesundheitsversorgung und-vorsorge für Kinder und Jugendliche
}

die Bedeutung dieses Faches gerade auf diesem Gebiet. Viele chronische Leiden des Erwachsenen könnten durch konsequente Nutzung des Wissens über Wachstum und Entwicklung zukünftig gelindert oder gar verhindert werden. In den Händen der Pädiatrie liegen die Konzepte und Chancen für gesünder aufwachsende zukünftige Generationen.

Die Erfolge in Diagnostik, Behandlung und Prävention von Gesundheitsstörungen gründen auf einer kontinuierlichen, verlässlich geförderten und gesicherten Forschungstätigkeit gerade an den universitären pädiatrischen Einrichtungen Gesellschaft und Staat sind gefordert, die Chancen moderner Kinder- und Jugendmedizin zum Wohle aller auch in Zukunft sicherzustellen. Dazu gehört nicht zuletzt die Berücksichtigung der Forschungstätigkeit auch in den Weiterbildungscurricula unseres Faches. Die auf einer Kalkulation der Durchschnittskosten beruhenden diagnosebezogenen Fallentgelte (DRG: „diagnosis related groups") sind unzureichend, um wissenschaftlich orientierte Pädiatrie zu refinanzieren. Infolgedessen wird es in Deutschland zunehmend schwieriger, exzellente Forschungsprojekte in der Pädiatrie zu etablieren. Um dem entgegenzuwirken, müssen Rahmenbedingungen geschaffen werden, die den Wert medizinischer Forschung stärker im Weiterbildungscurriculum betonen. Hierfür wird sich die DGKJ auch in $\mathrm{Zu}$ kunft einsetzen, denn in der Förderung des wissenschaftlichen Nachwuchses liegt das Potenzial für zukünftige medizinische Erfolge. Vor diesem Hintergrund sorgen wir für eine qualitativ hohe Weiterbildung u. a. mit der Monatsschrift Kinderheilkun$d e$, die neben Weiterbildungsthemen immer auch wissenschaftliche Originalarbeiten mit klinischer Relevanz publiziert. Darüber hinaus dienen der jährlich stattfindende Kinder- und Jugendärztekongress, seit 2004 die Repetitorien Pädiatrie und seit 2008 der pädiatrische Ernährungskurs der Fort- und Weiterbildung.

Kinder- und Jugendärzte/-innen waren und sind Promotoren für die Gesundheit und Lebensqualität künftiger Generationen. Gemeinsam können wir den hohen Versorgungsstandard der Kinder- und Jugendmedizin in Deutschland erhalten und weiterentwickeln. Ich möchte die Leser ermuntern, hierzu einen aktiven Beitrag als Mitglied der DGKJ zu leisten.

\section{br \\ F. Zepp \\ Präsident der DGKJ}

\section{Korrespondenzadresse}

Prof. Dr. F. Zepp

Zentrum für Kinder- und Jugendmedizin, Johannes Gutenberg-Universität Mainz Langenbeckstraße 1, 55131 Mainz zepp@kinder.klinik.uni-mainz.de 
Hier steht eine Anzeige.

算 Springer 